\title{
Full spectrum enhancement of the light harvesting efficiency of dye sensitized solar cells by including colloidal photonic crystal multilayers
}

\author{
A. Mihi, F. J. López-Alcaraz, and H. Míguez ${ }^{a)}$ \\ Instituto de Ciencia de Materiales de Sevilla, CSIC, Avenida Américo Vespúcio s/n, Isla de la Cartuja, \\ 41092 Sevilla, Spain
}

(Received 7 October 2005; accepted 21 March 2006; published online 9 May 2006)

\begin{abstract}
Herein we report a theoretical analysis of the efficiency of dye sensitized solar cells in which colloidal crystals are introduced in different configurations. We find that piling up different lattice constant crystals leads to light harvesting enhancement in the whole dye absorption range. We provide the optimum structural features of such photonic crystal multilayer needed to achieve a photocurrent efficiency enhancement of around $60 \%$ with respect to standard dye sensitized solar cells. We demonstrate that this improvement is the result of the optical absorption amplification effect of slow photon resonant modes partially confined within the absorbing part of the cell by the mirror behavior of the colloidal superlattice. (C) 2006 American Institute of Physics.
\end{abstract}

[DOI: 10.1063/1.2200746]

Dye sensitized solar cells (DSSCs) constitute an interesting alternative to solid state semiconductor photovoltaic devices, mainly due to their low cost of production. ${ }^{1,2}$ Briefly, a DSSC is made by a nanocrystalline titanium oxide layer doped with a ruthenium based dye supported on a conducting electrode and fully immersed in a redox electrolyte (typically an iodide solution). The whole system is sealed by a counterelectrode. The dye absorbs light in the visible range and injects the photogenerated electrons to the conduction band of the nanocrystalline titania, ${ }^{3}$ being the photoelectrons generated transferred to the supporting conducting substrate. At the other end of the circuit, the electrolyte is reduced at the counterelectrode and oxidized back after transferring an electron to the dye.

Even though current DSSC still shows lower efficiencies than the $p-n$ junction solar cells, there are many parameters to be investigated yet in order to improve light to current conversion. DSSC photovoltage can be increased by using other metal oxides such as $\mathrm{NbO}_{5}$ or $\mathrm{SrTiO}_{3}{ }^{4,5}$ Also higher photocurrents can be achieved by changing the sensitized dye, by combining cells with different dyes which absorb at different wavelength ranges or by means of introducing scattering structures that increase the matter-radiation interaction time, so the probability of photon absorption increases. ${ }^{6,7}$ Our work focuses on this latter approach. A modification in the photon collection of the cell affects the photogenerated current as explained in what follows. The light harvesting efficiency (LHE) or absorptance is the fraction of light intensity absorbed by the dye in the DSSC:

$$
\mathrm{LHE}=A=\frac{I_{\mathrm{abs}}}{I_{0}} .
$$

It depends on the extinction coefficient of the dye, its concentration, the thickness of the absorbing layer, and the time of flight of photons within that film. The incident photon to current conversion efficiency (IPCE) is proportional to the LHE and is given by the expression

\footnotetext{
${ }^{a)}$ Electronic mail: hernan@icmse.csic.es
}

$\mathrm{IPCE}=(\mathrm{LHE}) \Phi \eta$,

where $\Phi$ is the quantum yield of charge injection, and $\eta$ is the charge collecting efficiency by the glass supported electrode. From this magnitude its possible to obtain the generated photocurrent density in short-circuit $\left(J_{\mathrm{SC}}\right)$ by using the equation

$$
J_{\mathrm{sc}}=\int q \operatorname{IPCE}(\lambda) F(\lambda) d \lambda,
$$

where $q$ is the electron charge, and $F(\lambda)$ is the incident photon flux under AM 1.5 spectral irradiance. ${ }^{8}$ Its is clear from Eqs. (2) and (3) that increasing the amount of light absorbed by the dye (or LHE) leads to higher photocurrents. One option to improve the efficiency of a DSSC without substituting any of its components is to structure the materials; it is made of in such a way that the time of flight of photons through the absorbing material is enlarged, thus rising the probability of photogenerating electron-hole pairs. Some attempts in this line were performed adding polydisperse $\mathrm{TiO}_{2}$ particles at the dye sensitized $n c-\mathrm{TiO}_{2}$ layer. One step forward in this same direction but applying a different concept was given recently by Nishimura et al., ${ }^{9}$ who built the dye sensitized nc- $\mathrm{TiO}_{2}$ coating within a Grätzel solar cell in the form of a bilayer: One layer with the shape of a highly scattering photonic crystal $(\mathrm{PhC})$ structure, i.e., an inverse $\mathrm{TiO}_{2}$ opal; the other presents the form of a standard unstructured dyed nanocrystalline titania. This combined structure showed an enhancement of the photogenerated current efficiency of $26 \%$ when compared to a standard DSSC. As it has recently been proved, ${ }^{10}$ such enhancement comes from the localization of light within the unstructured dyed titania film due to the appearance of photon resonant modes. These resonances occur in systems composed by a photonic crystal possessing an overlayer deposited on top ${ }^{11}$ and at spectral regions in which the inverse opal is highly reflecting, i.e., within the photonic crystal pseudogap wavelength range.

Here we demonstrate that a colloidal crystal multilayer, employed as a highly scattering photonic structure combined with an absorbing dye-sensitized nc- $\mathrm{TiO}_{2}$ layer, leads to a large increase of the photogenerated current for the whole 


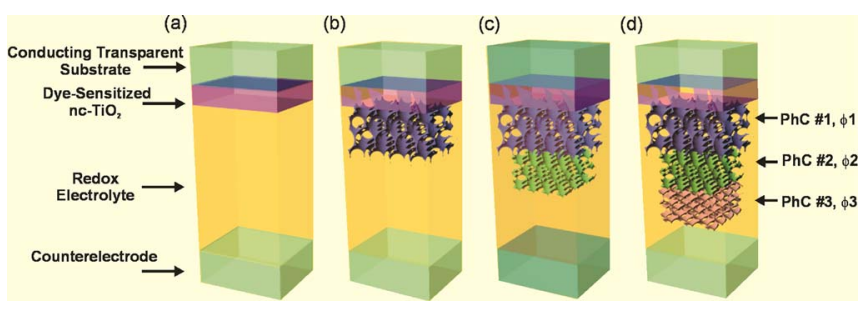

FIG. 1. (Color online) Schemes of the DSSCs whose LHEs are herein analyzed. (a) Standard DSSC made of a $\mathrm{nc}^{-\mathrm{TiO}_{2}}$ film followed by a bulk arranged titania slab of the same average refractive index and length of the compared PhC. (b) A photonic crystal based DSSC in which (b) a single inverse titania photonic crystal with sphere diameter $\phi_{1}$ (c), two inverse titania $\mathrm{PhC}$ multilayer with sphere diameters $\left(\phi_{1}, \phi_{2}\right)$, and (d) three inverse titania $\mathrm{PhC}$ multilayer with sphere diameters $\left(\phi_{1}, \phi_{2}\right.$, and $\left.\phi_{3}\right)$ of $17 \mathrm{ML}$ each, follow the nc- $\mathrm{TiO}_{2}$ film.

spectral region in which the dye absorbs. The effect of the stacking of colloidal lattices of different periodicity is to broaden the frequency range at which modes localized within the absorbing layer appear. A judicious choice of the lattice constants might allow one to achieve a $60 \%$ light harvesting efficiency enhancement with respect to a standard DSSC containing the same amount of absorbing material. Photonic crystal multilayers and superlattices are made of sequentially grown slabs of photonic colloidal crystals of different lattice parameters (i.e., different particle diameters). ${ }^{12}$

For our analysis of the optical properties of photonic crystal multilayer based DSSCs, we considered different stackings of three dimensional face centred cubic (fcc) lattices of interconnected spherical cavities whose walls were made of nc- $\mathrm{TiO}_{2}$, deposited onto an absorbing dye sensitized layer of $\mathrm{nc}^{-\mathrm{TiO}_{2}}$. The different structures analyzed in this work are schematized in Fig. 1. In all cases, light first impinges onto a conductive transparent substrate (with refractive index $n_{1}=1.45$ ), on which a $7 \mu \mathrm{m}$ thick film of ruthenium dye $\mathrm{N} 535$ sensitized nc- $\mathrm{TiO}_{2}$ has been deposited. The refractive index of this film, assuming that the electrolyte is filling all the mesopores, is $n_{2}=1.966$. The absorption coefficient of the ruthenium dye has been simulated as in Ref. 10. One, two, or three colloidal crystals are placed after the dyed nc- $\mathrm{TiO}_{2}$ layer, each one made of spherical shells of different inner diameters $\left(\phi_{1}, \phi_{2}\right.$, and $\left.\phi_{3}\right)$, as shown in Figs. 1(b)-1(d). The (111) planes of the crystals are parallel to the solar cell conducting substrate and therefore perpendicular to the incident light in our study. In all cases the refractive index of these nanocrystalline titania spherical shells is $n_{\text {shell }}=2.39$, a value that takes into account both the fact that titania synthesized in an opal from a liquid precursor is typically porous, as it has been experimentally reported, ${ }^{13}$ and that it must be infiltrated by the redox electrolyte. The number of close packed sphere monolayers composing the $\mathrm{PhCs}$ is arbitrarily fixed at $17 \mathrm{ML}$ (ML denotes monolayer), which is the thickness of the colloidal lattice grown in Ref. 9. Finally, the whole system is soaked in the TG 50 electrolyte, whose refractive index is 1.433 . For simplicity, the theoretical model considers the first conductive glass as the first media $\left(n_{1}=1.45\right)$ and the electrolyte as the last one $\left(n_{4}\right.$ $=1.433)$. In the configuration shown in Fig. 1(a), which we will use for comparison and refer to as the standard dye sensitized solar cell, the refractive index of the bulk porous $\mathrm{TiO}_{2}$ has been adjusted accordingly. $\mathrm{TiO}_{2}$ has been adjusted accordingly.
Downloaded 23 Feb 2010 to 161.111.180.191. Redistribution subject to AlP license or copyright; see http://apl.aip.org/apl/copyright.jsp

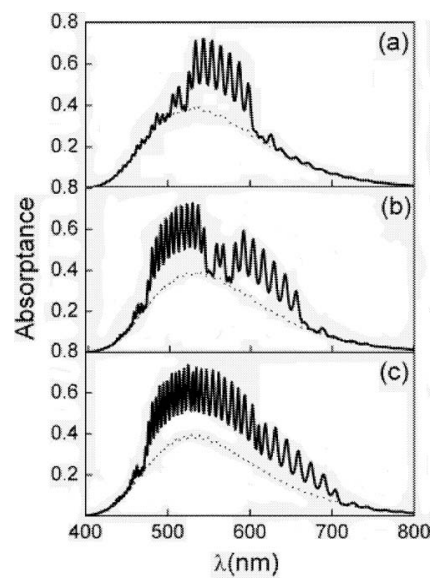

FIG. 2. Absorptance spectra of a dye sensitized solar cell involving an inverse titania photonic crystals slab $\left(\mathrm{LHE}_{\mathrm{PhC}}\right)$ of (a) one photonic colloidal crystal of $17 \mathrm{ML}$ and sphere diameter $\phi=202 \mathrm{~nm}$. (b) Two photonic crystals of $17 \mathrm{ML}$ each and with sphere diameters of $\phi_{1}=220 \mathrm{~nm}$ and $\phi_{2}=184 \mathrm{~nm}$. (c) Three photonic crystals of $17 \mathrm{ML}$ each and sphere diameters of $\phi_{1}$ $=234 \mathrm{~nm}, \phi_{2}=204 \mathrm{~nm}$, and $\phi_{3}=178 \mathrm{~nm}$. In all cases the absorptance spectra of the equivalent typical solar cell $\left(\mathrm{LHE}_{\text {standard }}\right)$ is also presented.

We seek to find the structural features of the cells depicted in Figs. 1(b)-1(d) that maximize the increment of photocurrent density $\Delta J_{\mathrm{SC}}$, defined as

$\Delta \mathrm{J}_{\mathrm{SC}}=\frac{\int\left[\operatorname{LHE}_{\mathrm{PhC}}(\lambda)\right] F(\lambda) d \lambda-\int\left[\operatorname{LHE}_{\text {standard }}(\lambda)\right] F(\lambda) d \lambda}{\int\left[\operatorname{LHE}_{\text {standard }}(\lambda)\right] F(\lambda) d \lambda}$,

where $\mathrm{LHE}_{\mathrm{PhC}}$ is the light harvesting efficiency or absorptance of a dye sensitized solar cell including photonic crystals, and $\mathrm{LHE}_{\text {standard }}$ refers to that of the standard DSSC. Both cells compared have the same amount of absorbing material and total width. LHE was calculated in each case using a transfer matrix formalism ${ }^{14}$ implemented in a MATLAB code, which allowed us to test a large number of structures in a reasonable time. To simplify, we neglect the vector nature of the radiation and consider just one possible direction of propagation, transforming our problem in a one dimensional (1D) scalar wave one. These assumptions have been repeatedly proven to yield correct results for three dimensional (3D) lattices, ${ }^{15}$ as long as only optical effects associated to lower bands are investigated and in a well-defined crystalline direction, as it happens in our case. A detailed discussion on the application of this type of calculations to simulate LHE in solar cells can be found elsewhere. ${ }^{10}$

As starting point, and in order to fix some relevant parameters, we have simulated the spectral response of the LHE of the dye sensitized solar cell described in Ref. 9, with some changes in the order of the components to maximize the efficiency. In Fig. 2(a) the absorptance of both the onephotonic-crystal based DSSC and the standard cell used for comparison are plotted. In this case, $\Delta J_{\mathrm{SC}}$ is maximized when the spherical cavity diameter was $\phi_{1}=202 \mathrm{~nm}$. The spectral behavior observed closely fits the experimental observations for the photogenerated current previously reported. ${ }^{9}$ With the above mentioned materials characteristics, increments in the photocurrent as large as $\Delta J_{\mathrm{SC}}=28 \%$ can be achieved, also in good quantitative agreement with 


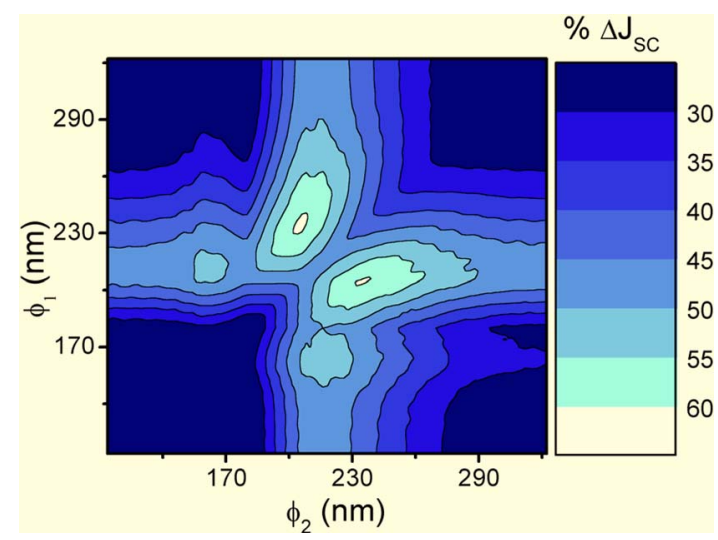

FIG. 3. (Color online) Photocurrent density enhancement map $\left(\% \Delta J_{\mathrm{SC}}\right)$ for a solar cell containing a dye-sensitized nanocrystalline titania film deposited on top of an inverse opal multilayer. The diameter of the bottom crystal has been fixed at $\phi_{3}=178 \mathrm{~nm}$, while the top and the middle crystal diameters $\left(\phi_{1}, \phi_{2}\right)$ are varied. All three lattices present a similar thickness of $17 \mathrm{ML}$.

of stacking a second and a third inverse titania opal with a different lattice parameters after the dye sensitized titania layer, as in Figs. 1(c) and 1(d), is to extend the wavelength range at which optical absorptance enhancement occurs, as can be clearly seen in Figs. 2(b) and 2(c). Widening the LHE amplification range yields a largely improved performance of the DSSC, a photocurrent increment of $\Delta J_{\mathrm{SC}}=48 \%$ being estimated for the optimized structure in the case of two inverse opals (spherical cavities with diameters $\phi_{1}=234 \mathrm{~nm}$, $\phi_{2}=204 \mathrm{~nm}$, and $\phi_{3}=178 \mathrm{~nm}$ ). With this latter structure it is possible to attain a colloidal crystal mirror able to provide LHE enhancement over most of the absorption range of the ruthenium dye. This structure is feasible and provides an extraordinary increase with respect to previously proposed ones. We have also carried out calculations including a larger number of photonic crystals in the DSSC. Only a slightly larger enhancement is achieved with respect to the three-opal stacking. Since almost all the absorption spectrum of the dye is already amplified with three crystals, hence the improvement would not justify the effort of building a more complex structure.

In order to visualize the dependence of $\Delta J_{\mathrm{SC}}$ with the lattice constants of the photonic crystal multilayer, we have plotted a two dimensional (2D) contour plot of $\Delta J_{\mathrm{SC}}$, keeping constant the diameter of the cavities in the bottom opal at its best value, $\phi_{3}=178 \mathrm{~nm}$, and varying $\phi_{1}$ and $\phi_{2}$. This photocurrent enhancement map is shown in Fig. 3. The abscissas correspond to the sphere diameter of the middle crystal $\left(\phi_{2}\right)$ and the ordinates to that of the cavities of the top crystal $\left(\phi_{1}\right)$, the one in contact with the absorbing layer. We can see that this map is almost symmetrical with respect to the $\phi_{1}=\phi_{2}$ line, indicating that, once a triplet of optimum diameters is found, the order in which the colloidal crystals are arranged does not substantially modify $\Delta J_{\mathrm{SC}}$. The inner shell diameters that maximize the photocurrent enhancement (up to $61 \%$ ) are $\phi_{1}=234 \mathrm{~nm}, \phi_{2}=204 \mathrm{~nm}$, fixed $\phi_{3}$
$=178 \mathrm{~nm}$. The enhancement rapidly decreases as one of the diameters gets too large or too small so the corresponding photonic crystal effect does not match the absorption range of the ruthenium dye any longer.

Enhancement of LHE takes place at wavelengths corresponding to the stop band of the photonic crystals involved. Such enhancement occurs at resonant modes which are partially localized within the absorbing dyed layer as a result of the mirrorlike behavior of the opals deposited behind it, as it has recently been shown by some of us. ${ }^{10}$ Partially confined photons travel slower through the absorbing layer and are more likely to be absorbed. Interestingly, the photonic crystals do not need to absorb, as it was originally assumed. ${ }^{9}$ If the presence of the anchored dye is also considered in the titania colloidal crystals, the simulated LHE increment would lead to lower $\Delta J_{\mathrm{SC}}$ than for the cases discussed above. It must be noticed that the absolute values of the photocurrent density are not necessarily lower in cells in which dyed opals are introduced, since the amount of the absorbing dye is also much larger. What our calculations prove is that a more efficient use of the material is done by placing nonabsorbing photonic crystals, since most of the light is harvested within the unstructured, dye sensitized $\mathrm{nc}-\mathrm{TiO}_{2}$ coating onto which the colloidal lattices are deposited.

To conclude, we have made a theoretical analysis of the performance of a dye sensitized solar cell in which inverse titania opal multilayers are included. We have found the optimum structural features to build the most efficient solar cells based on this approach. We foresee that this substantial improvement might not only lead to more competitive dye sensitized solar-to-electric energy conversion devices, but also find applications in related fields such as photocatalysis.

${ }^{1}$ B. O'Regan and M. Grätzel, Nature (London) 353, 737 (1991).

${ }^{2}$ M. Grätzel, Nature (London) 414, 338 (2001).

${ }^{3}$ J. Bisquert, D. Cahen, G. Hodes, S. Ruhle, and A. Zaban, J. Phys. Chem. B 108, 8106 (2004).

${ }^{4}$ K. Sayama, H. Sugihara, and H. Arakawa, Chem. Mater. 10, 3825 (1998).

${ }^{5}$ S. Burnside, J. E. Moser, K. Brooks, and M. Grätzel, J. Phys. Chem. B 103, 9328 (1999).

${ }^{6}$ A. Kay and M. Grätzel, Sol. Energy Mater. Sol. Cells 44, 99 (1996).

${ }^{7}$ Y. Tachibana, K. Hara, K. Sayama, and H. Arakawa, Chem. Mater. 14, 2527 (2002).

${ }^{8}$ Standard spectrum taken from http://rredc.nrel.gov

${ }^{9}$ S. Nishimura, V. Abrams, B. A. Lewis, L. Halaoui, T. E. Mallouk, K. D. Benkstein, J. Lagemaat, and A. J. Frank, J. Am. Chem. Soc. 125, 6306 (2003).

${ }^{10}$ A. Mihi and H. Míguez, J. Phys. Chem. B 109, 15968 (2005).

${ }^{11}$ A. Mihi, H. Míguez, I. Rodríguez, S. Rubio, and F. Meseguer, Phys. Rev. B 71, 125131 (2005).

${ }^{12}$ P. Jian, G. N. Ostojic, R. Narat, D. M. Mittleman, and V. L. Colvin, Adv. Mater. (Weinheim, Ger.) 13, 389 (2001).

${ }^{13}$ S. Nishimura, A. Shishido, N. Abrams, and T. E. Mallouk, Appl. Phys. Lett. 81, 4532 (2002).

${ }^{14}$ R. M. Bell, J. B. Pendry, L. Martín Moreno, and A. J. Ward, Comput. Phys. Commun. 85, 306 (1995).

${ }^{15}$ D. M. Mittleman, J. F. Bertone, P. Jiang, K. S. Hwang, and V. L. Colvin, J. Chem. Phys. 111, 345 (1999). 